\title{
Adrenalectomía laparoscópica bilateral sincrónica en feocromocitoma bilateral*
}

\author{
Drs. OCTAVIO A. CASTILLO C. ${ }^{1,2,3}$, RODRIGO CAMPOS P. ${ }^{1}$, RENE HENRÍQUEZ D. ${ }^{4}$, JUAN BRAVO P. ${ }^{4}$
}

1 Unidad de Urología, Clínica Indisa.

2 Facultad de Medicina, Universidad Andrés Bello.

${ }^{3}$ Facultad de Medicina, Universidad de Chile.

${ }^{4}$ Servicio de Urología, Hospital Regional de Talca. Chile.

\begin{abstract}
Bilateral laparoscopic synchronous adrenalectomy in bilateral pheochromocytoma: experience in 8 patients

Aim: To evaluate 8 cases of bilateral pheochromocytoma and laparoscopic synchronous treatment. Patients and Methods: From May 1999 to May 2010, 8 patients with bilateral pheochromocytoma were found. A complete preoperative workup was done. Results: We perform 8 transperitoneal laparoscopic bilateral synchronous adrenalectomy. There was no open converted procedure. Mean operative time was 184.37 min. (range 95 to $300 \mathrm{~min}$ ). Mean hospital stay was 3.8 days (range 3 to 5 days). Mean blood loss was $191.25 \mathrm{ml}$ (range 0 to $500 \mathrm{~min}$ ). In 7 cases the histopathological result was of pheochromocytoma and 1 as a malignant pheochomocytoma based on its histology. Conclusions: With very well establish diagnostic disease, an appropriate anesthetic strategy and as surgical skills, laparoscopic bilateral synchronic adrenalectomy is a feasible and safe surgical technique for bilateral pheochromocytomas.
\end{abstract}

Key words: Pheochromocytoma, bilateral adrenalectomy, laparoscopy.

\section{Resumen}

Introducción: La incidencia del feocromocitoma varía entre un 0,005 y un 0,1\% y de estos sólo un 10\% se presenta en forma bilateral. El objetivo de este trabajo es presentar nuestra experiencia en adrenalectomía laparoscópica bilateral sincrónica en pacientes con diagnóstico de feocromocitoma bilateral. Se revisa la literatura y se analizan nuestros resultados. Material y Métodos: Se realiza una revisión retrospectiva de todos los pacientes sometidos a una adrenelectomía laparoscópica bilateral sincrónica entre mayo de 1999 y mayo de 2010, con diagnóstico de un feocromocitoma bilateral. Todos los pacientes fueron estudiados con medición de catecolaminas plasmáticas y urinarias y estudio por imágenes. Resultados: Se realizaron 8 adrenelectomías laparoscópicas bilaterales sincrónicas. El tiempo promedio de cirugía fue de 184 min (rango 95-300 min). La estadía intrahospitalaria media fue de 3,8 días (rango de 3-5 días). El promedio de sangrado fue de $83 \mathrm{ml}$ (rango 0-500 ml). El diagnóstico histopatológico fue de feocromocitoma en todas las muestras, en un caso se

*Recibido el 9 de noviembre de 2010 y aceptado para publicación el 6 de marzo de 2011.

Correspondencia: Dr. Octavio A. Castillo C.

Av. Apoquindo 3990, Of. 809, Santiago, Chile.

C.P.: 7550112. Fax: (562) 2282524

octavio.castillo@indisa.cl 
diagnosticó un feocromocitoma maligno. No hubo conversión a cirugía abierta. Conclusiones: La adrenelectomía laparoscópica bilateral sincrónica para feocromocitomas bilaterales es un procedimiento seguro y de baja morbilidad, realizada por un cirujano con experiencia en cirugía laparoscópica. Es necesario tener previamente el diagnóstico de certeza para el manejo anestésico y postquirúrgico adecuado para estos pacientes.

Palabras clave: Feocromocitoma bilateral, adrenalectomía, laparoscopia.

\section{Introducción}

El Feocromocitoma (FC) es un tumor derivado de las células cromafines que producen y secretan catecolaminas. Más del $90 \%$ de los FC son de ubicación intra-abdominal, en médula suprarrenal o en localizaciones extra-adrenales (paragangliomas). $\mathrm{Su}$ incidencia oscila entre $0,005 \%$ y el $0,1 \%$ de la población adulta general y en un $0,1 \%$ a un $0,2 \%$ en la población con hipertensión arterial (HTA). La mayor incidencia es entre la $4^{\text {ta }}$ y $5^{\text {ta }}$ década de la vida con idéntica distribución según sexo ${ }^{1}$, 80-90\% se comportan como tumores benignos, a pesar de ello la presencia de FC se asocia a complicaciones como: infarto de miocardio, arritmias cardíacas, falla cardíaca, encefalopatía hipertensiva, accidentes cerebrovasculares y muerte súbita ${ }^{2-4}$. El 10\% de los FC son bilaterales, siendo este porcentaje mayor entre las presentaciones familiares o hereditarias. Entre un 10 a un $15 \%$ de los FC exhiben un comportamiento maligno; con el fin de diagnosticar un FC maligno se debe documentar invasión de órganos vecinos o enfermedad metastásica. Los sitios más frecuentes de metástasis son el hígado, pulmón y hueso (especialmente en columna, cráneo y costillas) ${ }^{5}$.

Aproximadamente el 10\% de los FC son hereditarios. Los tumores hereditarios son frecuentemente múltiples y bilaterales, rara vez malignos. Los síndromes hereditarios asociados a FC incluyen a la Neoplasia Endócrina Múltiple tipo 2 (NEM 2), Enfermedad de Von Hippel-Lindau (VHL) y las displasias neuro ectodérmicas que consisten en neurofibromatosis, esclerosis tuberosa y síndrome de Sturge-Weber ${ }^{5}$.

No cabe duda que el tratamiento quirúrgico constituye la solución definitiva para los FC. Desde la descripción inicial por Gagner en 1992 ${ }^{6}$, la adrenalectomía laparoscópica se ha convertido en la cirugía de elección para la glándula suprarrenal. Muchos artículos publicados desde entonces han confirmado las ventajas de la técnica en cuanto a menor estadía hospitalaria, menor requerimiento de analgésicos y rápida reincorporación a la actividad normal, además de evitar las complicaciones propias de las incisiones tradicionales ${ }^{7-9}$.

A pesar de que el abordaje laparoscópico bilateral simultáneo, puede aumentar el tiempo operatorio y la morbilidad con respecto a procedimientos unilaterales ${ }^{10}$, en la actualidad está reportado en la bibliografía con resultados satisfactorios en tumores bilaterales (incluyendo FC y otros tumores suprarrenales), tanto en resecciones totales como parciales de la glándula suprarrenal ${ }^{10-22}$.

El objetivo de este trabajo es presentar nuestra experiencia en adrenalectomía laparoscópica sincrónica en pacientes con diagnóstico de feocromocitoma bilateral, se analizan resultados, complicaciones y seguimiento post-operatorio.

\section{Material y Método}

Se realiza una revisión retrospectiva de todos los pacientes sometidos a una adrenalectomía laparoscópica sincrónica por un feocromocitoma bilateral en nuestra unidad en el período comprendido entre mayo de 1999 y mayo de 2010. Todos los pacientes fueron estudiados por presentar una hipertensión severa y fueron diagnosticados, previo a la cirugía, con medición de catecolaminas plasmáticas y urinarias, y estudio por imágenes con una Tomografía Axial Computada (TAC) abdominal (Figura 1), o una Resonancia Magnética (RM) según las características de las lesiones.

En todos los pacientes se realizó una adrenalectomía laparoscópica bilateral sincrónica por vía transperitoneal según técnica publicada previamente por Castilho et $\mathrm{al}^{21}$. Previo a la cirugía los pacientes fueron alfa y beta bloqueados.

Las variables analizadas fueron: edad, sexo, patología asociada, tiempo quirúrgico, complicaciones, sangrado intraoperatorio, conversión, necesidad de transfusión, tiempo de estadía hospitalaria, resultados histopatológicos y evolución a largo plazo.

El seguimiento post operatorio a largo plazo se realizó con una evaluación clínica, determinaciones urinarias y séricas de catecolaminas, así como métodos de diagnóstico por imágenes.

Se realizó, además, una completa revisión de la bibliografía relacionada con el tema.

\section{Resultados}

Se realizó una adrenalectomía laparoscópica bilateral sincrónica en 8 pacientes en el período antes descrito, lo cual corresponde al 2,5\% de una serie personal de 315 adrenalectomías laparoscópicas, 


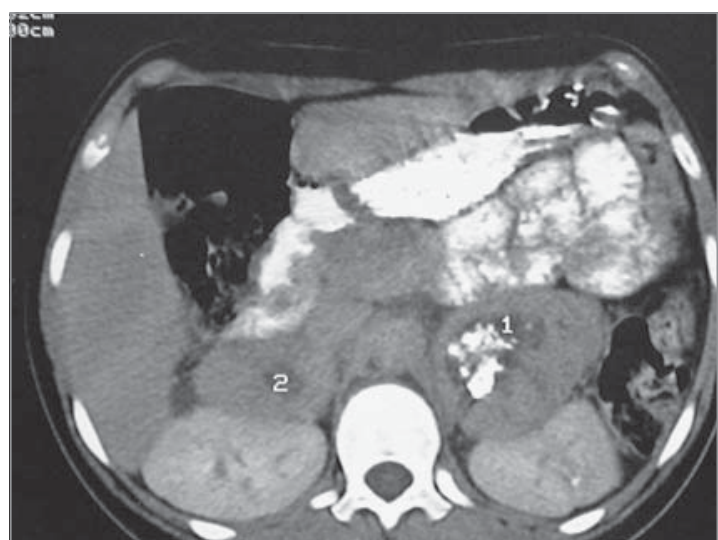

Figura 1. Tomografía computada que muestra feocromocitoma bilateral.

realizadas en el período de 1994 al 2010. La edad promedio fue de 32,7 años (16 a 55 años). El 62,5\% fueron mujeres. La clínica que motivó la consulta fue hipertensión severa en los 8 casos. En un paciente se presentó asociado a un síndrome de Van Hippel-Lindau, un paciente tenía el antecedente de feocromocitoma familiar, y dos pacientes tenían una NEM 2 (uno de los cuales presentó un cáncer medular de tiroides). En el resto de los pacientes no se encontraron enfermedades hereditarias. En la Tabla 1 pueden observarse las características demográficas de la población.

No hubo complicaciones quirúrgicas así como tampoco necesidad de conversión a cirugía abierta. Cinco pacientes fueron sometidos a una adrenalectomía total laparoscópica bilateral (Figuras 2 y 3) y en 3 pacientes se realizó una adrenalectomía unilateral con adrenalectomía parcial de la glándula contralateral, sin clipaje de la vena adrenal. El tiempo promedio de la cirugía bilateral fue de 184,37 min (95-300 min). Si tenemos en cuenta el tiempo de resección de cada glándula, en el lado izquierdo el tiempo promedio fue de 96,8 min (40-150 min) y del lado derecho fue de 87,5 min (45-180 min). El sangrado promedio fue de $83 \mathrm{ml}(0-500 \mathrm{ml})$, ningún paciente requirió transfusión (Figura 4). El diagnóstico histopatológico fue de un feocromocitoma bilateral en todos los casos, y en un paciente se hizo el diagnóstico de un feocromocitoma maligno; aún cuando no existen criterios histológicos claros (invasión vascular, invasión capsular y pleomorfismo) y sólo el comportamiento cínico define su malignidad. Con una mediana de seguimiento de 91 meses, seis pacientes se encuentran en tratamiento corticoidal suplementario, dos pacientes a quienes se les realizó

Tabla 1. Características demográficas de la población

\begin{tabular}{|c|c|c|c|c|c|c|c|c|c|c|c|}
\hline $\mathbf{n}$ & Edad & Sexo & $\begin{array}{l}\text { Riesgo } \\
\text { (ASA) }\end{array}$ & $\begin{array}{l}\text { Patología } \\
\text { asociada }\end{array}$ & Lado & Operación & $\begin{array}{c}\text { Tiempo } \\
\text { Op. } \\
\text { (min) }\end{array}$ & $\begin{array}{c}\text { Tiempo } \\
\text { hosp. } \\
\text { (días) }\end{array}$ & $\begin{array}{l}\text { Compli- } \\
\text { caciones }\end{array}$ & $\begin{array}{c}\text { Sangrado } \\
\text { Intra-op. } \\
\text { (MI) }\end{array}$ & $\begin{array}{c}\text { Con- } \\
\text { versión }\end{array}$ \\
\hline \multirow[t]{2}{*}{1} & 35 & $\mathrm{~F}$ & I & \multirow{4}{*}{$\begin{array}{l}\text { HTA } \\
\text { Von-Hippel } \\
\text { Lindau } \\
\text { HTA }\end{array}$} & Der & \multirow[t]{2}{*}{ Extirpación } & 180 & 5 & \multirow[t]{2}{*}{ No } & 100 & \multirow[t]{2}{*}{ No } \\
\hline & & & & & Izq & & 120 & 5 & & 0 & \\
\hline \multirow[t]{2}{*}{2} & 34 & $\mathrm{~F}$ & II & & Der & Extirpación & 150 & 5 & \multirow[t]{2}{*}{ No } & 300 & \multirow[t]{2}{*}{ No } \\
\hline & & & & & Izq & & 150 & 5 & & 100 & \\
\hline \multirow[t]{2}{*}{3} & 19 & M & II & \multirow{2}{*}{$\begin{array}{l}\text { HTA } \\
\text { Feo familiar }\end{array}$} & Izq & Tumorectomía & 75 & 4 & \multirow[t]{2}{*}{ No } & 50 & \multirow[t]{2}{*}{ No } \\
\hline & & & & & Der & Extirpación & 70 & 4 & & 20 & \\
\hline \multirow[t]{2}{*}{4} & 28 & F & II & \multirow[t]{2}{*}{ HTA } & Der & Extirpación & 60 & 3 & \multirow[t]{2}{*}{ No } & 20 & \multirow[t]{2}{*}{ No } \\
\hline & & & & & Izq & & 80 & 3 & & 40 & \\
\hline \multirow[t]{2}{*}{5} & 31 & $\mathrm{~F}$ & II & \multirow{4}{*}{$\begin{array}{l}\text { HTA } \\
\text { Ca tiroides } \\
\text { MEN } 2 \\
\text { HTA }\end{array}$} & Izq & Extirpación & 140 & 3 & \multirow[t]{2}{*}{ No } & 500 & \multirow[t]{2}{*}{ No } \\
\hline & & & & & Der & Tumorectomía & 45 & 3 & & 50 & \\
\hline \multirow[t]{2}{*}{6} & 55 & M & II & & Der & Extirpación & 45 & 3 & \multirow[t]{2}{*}{ No } & 50 & \multirow[t]{2}{*}{ No } \\
\hline & & & & & Izq & & 50 & 3 & & 50 & \\
\hline \multirow[t]{2}{*}{7} & 44 & M & II & HTA & Izq & Extirpación & 70 & 5 & \multirow[t]{2}{*}{ No } & 0 & \multirow[t]{2}{*}{ No } \\
\hline & & & & $\mathrm{M}$ & Der & Tumorectomía & 50 & 5 & & 0 & \\
\hline \multirow[t]{2}{*}{8} & 16 & $\mathrm{~F}$ & II & HTA & Der & Extirpación & 100 & 3 & \multirow[t]{2}{*}{ No } & 150 & \multirow[t]{2}{*}{ No } \\
\hline & & & & & Izq & & 90 & 3 & & 100 & \\
\hline
\end{tabular}




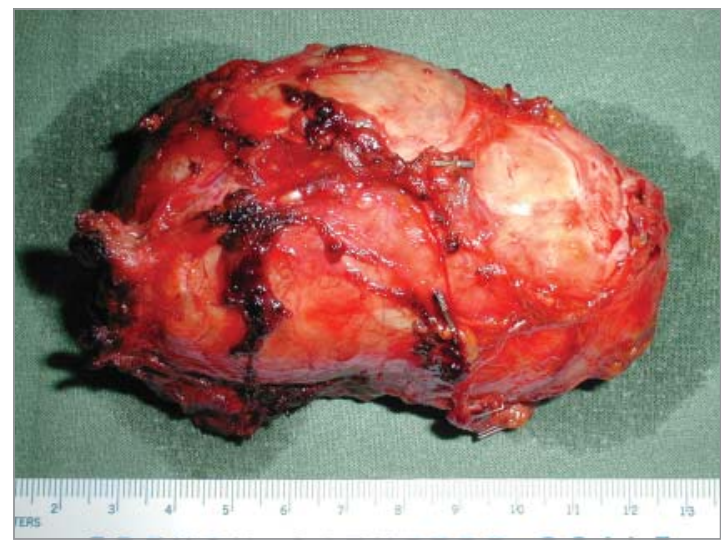

Figura 2. Lesión adrenal izquierda de $10 \mathrm{~cm}$.

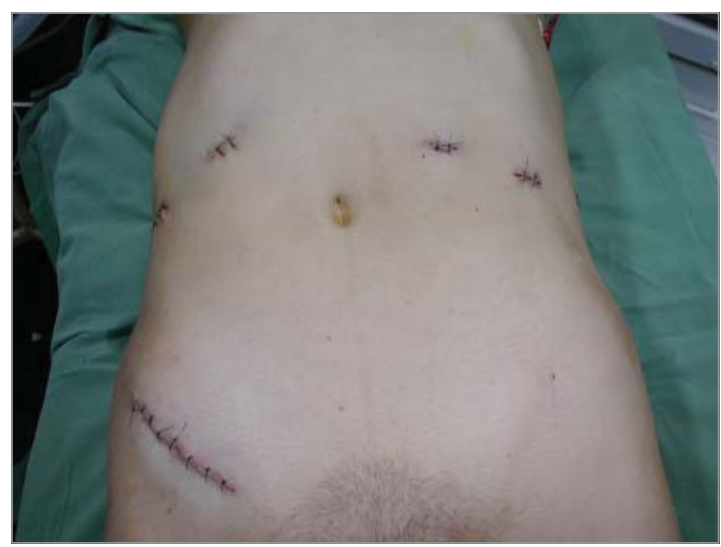

Figura 4. Aspecto final de la cirugía, con incisión baja a derecha para extracción de ambos tumores adrenales.

una tumorectomía no tienen necesidad de tratamiento suplementario. El paciente con el feocromocitoma maligno no ha presentado recidiva tumoral durante el seguimiento.

\section{Discusión}

Se estima que por año se presentan de 1 a 2 casos nuevos de FC por millón de habitantes ${ }^{28}$, de ellos, sólo el 10\% presentan bilateralidad (aunque el caso de FC bilateral puede presentarse en $80 \%$ de pacientes con mutación genética o $\mathrm{FC}$ hereditario ${ }^{29}$ ). Nuestra serie muestra pacientes con FC bilateral donde el factor hereditario estuvo presente en el $50 \%$, lo que apoya la idea de que ante la presencia de enfermedad adrenal (FC) bilateral, se debe tratar de identificar patología hereditaria mediante los métodos diagnósticos correspondientes.

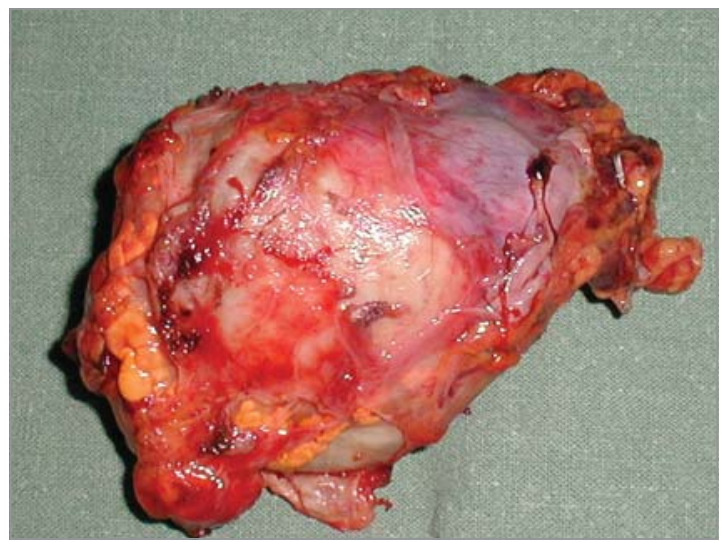

Figura 3. Lesión adrenal derecha de $8 \mathrm{~cm}$.

En la serie analizada, un caso (12,5\%) fue catalogado por su histopatología como un FC maligno (similar al 10\% de malignidad reportado en la bibliografía ${ }^{5}$ ), aunque no se ha detectado metástasis en dicho paciente hasta el momento.

Inicialmente la presencia de FC fue considerada una contraindicación para laparoscopía debido a reportes que mencionaban que el neumoperitoneo podría promover liberación aguda de catecolaminas $^{21}$; sin embargo, varios autores han demostrado que cuando existe una adecuada preparación preoperatoria, los cambios hemodinámicos son leves y bien tolerados por los pacientes s,6,9,19,21 $^{\text {. Si tenemos }}$ en cuenta esto y lo referido a las claras ventajas de la laparoscopía inherentes al método ${ }^{7-9}$, sobre todo cuando se trata del tratamiento de patología adrenal $^{19,27}$, está claro que el tratamiento laparoscópico de feocromocitomas suprarrenales debería ser el gold standard en la actualidad; aunque a la fecha no hemos encontrado publicaciones de grandes series prospectivas-randomizadas, comparando adrenalectomía laparoscópica vs cirugía convencional.

El aumento de la incidencia de patología adrenal bilateral ha creado un dilema en cuanto al tratamiento óptimo de la glándula contralateral. Las estrategias de tratamiento incluyen adrenalectomía total bilateral sincrónica, adrenalectomía subtotal ${ }^{23}$ y/o una adrenalectomía metacrónica. En la Tabla 2 se encuentran resumidas las mayores series de adrenalectomías laparoscópicas bilaterales sincrónicas reportadas hasta la fecha, observándola se pone de manifiesto que el enfoque bilateral sincrónico es factible y ha ido ganando adeptos con el transcurrir del tiempo. Takata ${ }^{15}$, en su artículo revisó 12 series publicadas de adrenalectomías laparoscópicas bilaterales totales, con al menos cinco casos en cada una, mostrando un tiempo quirúrgico promedio de 288 minutos, con una tasa de conversión de 4,7\% 
ADRENALECTOMÍA LAPAROSCÓPICA BILATERAL SINCRÓNICA EN FEOCROMOCITOMA BILATERAL

Tabla 2. Mayores series de adrenalectomías laparoscópicas bilaterales sincrónicas

\begin{tabular}{|c|c|c|c|c|c|}
\hline Serie (año) & Diagnóstico (n) & $\begin{array}{c}\text { Tasa de } \\
\text { conversión (\%) }\end{array}$ & $\begin{array}{c}\text { Tipo de } \\
\text { procedimiento }\end{array}$ & $\begin{array}{c}\text { Tiempo } \\
\text { quirúrgico, min }\end{array}$ & $\begin{array}{c}\text { Pérdida } \\
\text { de sangre, } \mathrm{mL}\end{array}$ \\
\hline Bonjer et al (2000) & SC (13), FC (3) & 6 & AT (R) & 214 & 121 \\
\hline Vella et al (2001) & SC (19) & 16 & AT & 252 & ND \\
\hline Hawn et al (2002) & SC (18) & 0 & AT & 296 & 218 \\
\hline Lihara et al (2003) & FC (7), SC (2) & 0 & $\begin{array}{l}\mathrm{AT}(4), \mathrm{AP}(4) . \\
\mathrm{AT}+\mathrm{AP}(1)\end{array}$ & ND & ND \\
\hline Jäger et al (2004) & SC (16), FC (2) & 0 & $\mathrm{AT}(16 \mathrm{~T}, 2 \mathrm{R})$ & 289 & 125 \\
\hline Porpiglia et al (2004) & SC (13) & 15 & $\mathrm{AT}$ & 234 & 340 \\
\hline Diner et al (2005) & FC (10) & 0 & AP & 187 (cada lado) & 100 \\
\hline Walz et al (2006) & FC (13), SC (27) & 5 & $\begin{array}{l}\text { AT (25), AP (10), } \\
\text { AT + AP (5); (R) }\end{array}$ & ND & ND \\
\hline Castillo et al (2007) & $\begin{array}{l}\mathrm{FC}(6), \mathrm{SC}(6), \\
\operatorname{APr}(2), \mathrm{TNF}(6)\end{array}$ & 0 & $\begin{array}{l}\mathrm{AT}(15), \mathrm{AP}(2), \\
\mathrm{AT}+\mathrm{AP}(3)\end{array}$ & 79,2 (cada lado) & 65,4 \\
\hline Takata et al (2008) & SC (25), FC (5) & 0 & $\mathrm{AT}$ & 290 & 81 \\
\hline Chow et al (2008) & SC (68) & 13 & AT & ND & ND \\
\hline Liao et al (2009) & SC (7), APr (5) & 0 & AT (6), AP (6) & 390 (AP), 255 (AT) & 79 \\
\hline
\end{tabular}

SC, Síndrome de Cushing; FC, Feocromocitoma; TNF, Tumor no funcionante; APr, Aldosteronismo primario; AT, Adrenalectomía Total; AP, Adrenalectomía Parcial; T, Transperitoneal; R, Retroperitoneoscopía; ND, No disponible.

y una tasa de complicaciones del 13\%. En nuestra serie tenemos menores tiempos quirúrgicos, no hay conversiones ni complicaciones intraoperatorias. La tabla además permite observar bajo número de casos de FC, lo que se condice con la baja incidencia de la patología en cuestión.

La mayoría de los autores coinciden que la adrenalectomía bilateral sincrónica está indicada si la enfermedad es bilateral al momento del diagnóstico. La cirugía bilateral simultánea es un abordaje bien tolerado, preferido para evitar terapia prolongada con alfa bloqueantes, así como múltiples inducciones anestésicas, en las cuales el riesgo de hipertensión persiste.

Aunque en nuestra serie sólo se usó la técnica transperitoneal, el procedimiento de adrenalectomía ha sido descrito y con buenos resultados por acceso retroperitonal, Thomas y Gill ${ }^{10}$, comparan el acceso retroperitoneal con el transperitoneal, mencionando la seguridad del procedimiento bilateral, la mínima morbilidad y afirman que ambos accesos no muestran diferencia significativa respecto al tiempo quirúrgico, pérdida sanguínea, estancia hospitalaria y requerimiento de analgesia. Hemal y cols ${ }^{26}$, mencionan la facilidad de la retroperitoneoscopía para acceder a la glándula adrenal, la mínima movilización del colon y además de que es un acceso viable en pacientes con cirugías abdominales previas, aunque el acceso por retroperitonescopía exige también el cambio de posición de lumbotomía, al igual que la técnica transperitoneal.

En un intento de preservar la función cortical adrenal, tratando de evitar la terapia de reemplazo con corticoides, algunos centros prefieren la adrenalectomía subtotal ${ }^{17,20,22}$. Además no se sabe con certeza la cantidad de tejido adrenal residual necesario para conservar una función adecuada. Brauckhoff y $\operatorname{cols}^{24,25}$, reportaron 14 pacientes a los que se les realizó adrenalectomía subtotal con ligadura y sección de la vena principal. La prueba de ACTH confirmó función adreno-cortical satisfactoria. Concluyeron que la vena principal no parece ser crucial para mantener función y que 1/3 de la glándula debería ser suficiente. En nuestro caso de los tres pacientes a quienes se conservó glándula suprarrenal, 2 permanecen sin terapia de reemplazo.

A modo de conclusión podemos afirmar que la adrenalectomía laparoscópica bilateral sincrónica para feocromocitomas bilaterales, es un procedimiento seguro y de baja morbilidad, realizada por un cirujano con experiencia en cirugía laparoscópica. Es necesario tener previamente el diagnóstico de certeza para el manejo anestésico y postquirúrgico adecuado para estos pacientes. 


\section{Referencias}

1. Karagiannis A, Mikhailidis DP, Athyros VG, Harsoulis F. Pheocromocytoma: an update on genetics and management. Endocr Relat Cancer 2007;14:935-56.

2. Schurmeyer TH, Engeroff B, Dralle H, Von zurMuhlen A. Cardiological effects of catecholamine-secreting tumors. Eur J Clin Invest. 1997;27:189-95.

3. Liao WB, Liu CF, Chiang CW, Kung CT, Lee CW. Cardiovascular manifestations of pheochromocytoma. Am J Emerg Med. 2000;18:622-5.

4. Brouwers FM, Lenders JW, Eisenhofer G, Pacak K. Pheochromocytoma as an endocrine emergency. Rev Endocr Metab Disord. 2003;4:121-8.

5. Mittendorf BA, Evans DB, Lee JE, Perrier ND. Pheochromocytoma: advances in genetics, diagnosis, localization and treatment. Hematol Oncol Clin North Am. 2007:21;509-25.

6. Gagner M, Lacroix A, Bolte E. Laparoscopic Adrenalectomy in Cushing's Syndrome and pheochromocytoma. N England J Med. 1992;327:1033.

7. Korman JE, Ho T, Hiatt JR, Phillips EH. Comparison of laparoscopic and open adrenalectomy. Am Surg. 1997;63:908-12.

8. MacGillivray DC, Shichman SJ, Ferrer FA, Malchoff CD. A comparison of open vs laparoscopic adrenalectomy. Surg Endosc. 1996;10:987-90.

9. Smith CD, Weber CJ, Amerson JR. Laparoscopic adrenalectomy: New gold standard. World J Surg. 1999;23:389-96.

10. Thomas HS, Inderbir SG. Bilateral Laparoscopic Adrenalectomy: retroperitoneal and transperitoneal approaches. Urology 2002;59:184-9.

11. Fernández-Cruz L, Sáenz A, Benarroch G, Sabater L, Taurá P. Total bilateral laparoscopic adrenalectomy in patients with Cushing's syndrome and multiple endocrine neoplasia (IIa). Surg Endosc. 1997;11:103-7.

12. Hawn MT, Cook D, Deveney C, Sheppard BC. Quality of life after laparoscopic bilateral adrenalectomy for Cushing's disease. Surgery 2002;132;1064-9.

13. Jäger F, Heintz A, Junginger T. Synchronous bilateral endoscopic adrenalectomy: experiences after 18 operations. Surg Endosc. 2004;18:314-8.

14. Porpiglia F, Fiori C, Bovio S, Destefanis P, Ali A, Terrone C, et al. Bilateral adrenalectomy for Cushing's syndrome: a comparison between laparoscopy and open surgery. J Endocrinol Invest. 2004;27:654-8.

15. Takata MC, Kebebew E, Clark OH, Duh QY. Laparoscopic bilateral adrenalectomy: results for 30 consecutive cases. Surg Endosc. 2008;22:202-7.

16. Chow JT, Thompson GB, Grant CS, Farley DR, Richards ML, Young WF Jr. Bilateral laparoscopic adrenalectomy for corticotrophin-dependent Cushing's syndrome: a review of the Mayo Clinic experience. Clin Endocrinol (Oxf). 2008;68:513-9.

17. Diner EK, Franks ME, Behari A, Linehan WM, Walther MM. Partial adrenalectomy: the National Cancer Institute experience. Urology 2005;66:19-23.

18. Walz MK, Alesina PF, Wenger FA, Deligiannis A, Szuczik A, Petersenn S, et al. Posterior retroperitoneoscopic adrenalectomy results of 560 procedures in 520 patients. Surgery 2006;140:943-50.

19. Castillo O, Vitagliano G, Cortés O, Kerkebe M, Pinto I, Arellano L. Bilateral laparoscopic adrenalectomy. J Endourol. 2007;21:1053-8.

20. Lihara M, Suzuki R, Kawamata A, Omi Y, Kodama H, Igari $\mathrm{Y}$, et al. Adrenal-preserving laparoscopic surgery in selected patients with bilateral adrenal tumors. Surgery 2003;134: 1066-73.

21. Castilho LN, Simoes FA, Santos AM, Rodrigues TM. Pheocromocytoma: A Long term Follow-up of 24 patients undergoing laparoscopic adrenalectomy. Int Braz J Urol. 2009;35:24-35.

22. Chun-Hou Liao, Shiu-Dong Chung, Ming-Kuen Lai, Hong-Jeng Yu, Shih-ChiehChueh. Laparoscopic simultaneous bilateral partial and total adrenalectomy: a longer follow-up. BJU International 2009;104:126973.

23. Walther MM, Herring J, Choyke PL, Linehan WM. Laparoscopic Partial Adrenalectomy in patients with Hereditary forms of pheochromocytoma. J Urol. 2000; 164:14-7.

24. Brauckhoff M, Gimm O, Thanh PN, Bar A, Ukkat J, Brauckhoff $\mathrm{K}$, et al. Critical size of residual adrenal tissue and recovery from impaired early postoperative adrenocortical function after subtotal bilateral adrenalectomy. Surgery 2003;134:1020-7.

25. Brauckhoff M, Stock K, Stock S, Lorenz K, Sekulla C, Brauckhoff K, et al. Limitations of intraoperative adrenal remnant volume measurement in patients undergoing subtotal adrenalectomy. World J Surg. 2008;32;863-72.

26. Hemal AK, Kumar R, Misra MC, Gupta NP, Chumber S. Retroperitoneoscopic adrenalectomy for pheochromocytoma: comparison with open surgery. JSLS 2003;7:341-5.

27. Castillo O, Cortés O, Kerkebe M, Pinto I, Arellano L, Contreras M. Cirugía laparoscópica en el tratamiento de enfermedades adrenales: experiencia en 200 casos. Actas Urol Esp. 2006;30:926-32.

28. Elder EE, Elder G, Larsson C. Pheochromocytoma and functional paraganglioma syndrome: no longer the $10 \%$ tumor. J Surg Oncol. 2005;89:193-201.

29. Mirallie E, Cariou B, Kraeber-Bodere F. Bilateral pheochromocytoma. Genetics and treatment. Ann Chir. 2005;130:273-6. 